\title{
(Re)produciendo profesionales peruanos: la asistencia social y la ciudadanía-materna de las madres quechuas empobrecidas
}

\section{Rebecca Irons}

University College London

rebecca.irons.14@ucl.ac.uk

\section{RESUMEN}

A través de los discursos de independencia financiera y «profesionalización» de la descendencia promovidos por la asistencia médica (SIS) y social (Juntos) provista por el Estado, las mujeres quechuas que viven en la pobreza descubren que, al ingresar a la maternidad, su plena ciudadanía se vuelve condicional a los comportamientos exitosos y la administración de los hijos para obtener un medio de vida más «deseable» que el suyo. Esto sugiere que la maternidad, mientras se es pobre, da un valor moral a las mujeres que el Estado usa para justificar su monitoreo y gobierno.

Este artículo se basa en un año de trabajo de campo etnográfico en comunidades rurales y centros o puestos de salud en la provincia de Vilcashuamán, departamento de Ayacucho, Perú. Se realizaron cien entrevistas con mujeres, hombres y trabajadores de la salud, además de una importante observación participante.

Si bien los discursos generalizados sobrecargan las libertades de las mujeres, existen alternativas a la «profesionalización» de los jóvenes indigenas que no requieren la educación financiera impuesta actualmente a las madres. Este documento sugiere que los valores morales asignados a las maternidades pobres a veces se usan injustamente comojustificación para la intervención reproductiva y la revocación de la ciudadanía plena para las mujeres indígenas pobres.

Palabras clave: maternidad, salud, ciudadanía, asistencia social, planificación familiar. 


\title{
(Re)Producing Peruvian Professionals: State Welfare and Poor Que- chua Mother's Maternal Citizenship
}

\author{
ABSTRACT
}

Through the discourses of financial-independence and "professionalisation" of offspring promoted by state-provided health-care and welfare (Juntos), Quechua women living in poverty find that upon entering motherhood their full citizenship becomes conditional on successful behaviours and stewardship of children to a more "desirable" livelihood than their own. This suggests that motheringwhile-poor places a moral value on women that the state uses to justify monitoring and governing them.

This paper is based on one-year's ethnographic-fieldwork in rural communities and health centres or posts in Vilcashuaman province, Ayacucho department, Peru. 100 interviews were conducted with women, men and health-workers, in addition to substantial participant-observation.

Whilst the pervasive discourses overburden women's freedoms, there are alternatives to "professionalization» of indigenous-youth that do not require the financial literacy currently imposed on mothers. This paper suggests that moral values placed on poor-maternities are sometimes used unfairly as a justification for reproductiveintervention and revocation of full-citizenship for poor, indigenous women.

Keywords: motherhood, health, citizenship, state welfare, family planning. 


\section{INTRODUCCIÓN}

El Estado peruano considera que las familias pobres — y en particular las madres están «en riesgo», categoría cargada de valor negativo y que también justifica la intervención gubernamental. Aquellos que califican y disfrutan del Seguro Integral de Salud (SIS) gratuito del gobierno forman parte de esta población en riesgo. Como señala Bridges sobre las mujeres pobres en los Estados Unidos, su estado de riesgo está respaldado por su búsqueda de atención médica subsidiada por el Estado. Las presunciones del «riesgo» de sus cuerpos y vidas se confirman por su presencia en la clínica (2011, p. 167). Desde este punto de vista, aquellos que están en riesgo debido a su pobreza también tienen cuerpos riesgosos y rebeldes (p. 16), especialmente cuando se abordan sus capacidades reproductivas supuestamente fuera de control. El factor de riesgo ayuda a justificar la intervención estatal en los cuerpos reproductivos de las mujeres más pobres (tales justificaciones se usaron en las esterilizaciones anteriores, bajo Fujimori, por ejemplo) (Ballón, 2014), principalmente a través del Programa de Planificación Familiar (PPFF) del gobierno, ejecutado a través del Ministerio de Salud (Minsa).

Sin embargo, abordar a las madres con riesgo no se detiene allí: otros programas estatales de asistencia social también se dirigen a esta población. Por ejemplo, el programa Juntos (que se analiza a continuación) también se desarrolló para poblaciones «en riesgo» como una forma de aliviar su pobreza a través de las finanzas (Meltzer, 2013, p. 643). Sin embargo, el elemento de riesgo inherente a estas poblaciones no es simplemente que son pobres, sino que son madres pobres. Han optado por la decisión supuestamente irracional de tener hijos mientras son pobres - «la decisión de reproducirse mientras son pobres irracional e irresponsable»-, tomada por mujeres que permiten que su «pobreza entrecruce con la maternidad» (Bridges, 2017, p. 109) y, por lo tanto, deben ser vigiladas por el Estado. Esto supuestamente justifica la intervención estatal y el control sobre sus vidas. No se puede confiar en ellas. Además, como se argumentará en 
este documento, debido a que la toma de decisiones tan irresponsable las llevó a quedar embarazadas mientras eran pobres, tienen su reclamo de ciudadanía plena revocado hasta el momento en que puedan dar a luz a niños competentes y profesionales que sean étnicos culturalmente y racialmente superiores a su madre indigente, en opinión del Estado.

De hecho, este es un discurso frecuente, aunque no necesariamente correcto. Como Yon Leau (2000) muestra en su investigación sobre la planificación familiar entre los quechuas, las mujeres a menudo solo quieren dos hijos en total, lo cual no es un deseo inflado para hacerlas «arriesgadas». Además, las mujeres quechuas y aimaras informaron deseos similares para familias más pequeñas en otros informes en ese momento. Por ejemplo, ver Ancash (Calisaya, 2004); Ayacucho (Salcedo, 2004); Huancavelica (Condori, 2003); Puno (Estrada Alarcón, 2004). Además, también se debe tener en cuenta que ni FP bajo SIS, ni Juntos, realmente requieren que la mujer sea la principal receptora de servicios o asistencia social (los hombres pueden acceder a la anticoncepción: Juntos solo requiere un «representante del hogar» (Meltzer, 2013, p. 648). Por lo tanto, a pesar de sus supuestos reclamos de empoderar y atender las necesidades de las mujeres «arriesgadas», estos programas pueden terminar afianzando potencialmente los «roles de género tradicionales: el papel de las mujeres como madres» (Meltzer, 2013, p. 648).

Esto puede volverse particularmente problemático cuando la pobreza se cruza con el origen étnico, como es el caso de las mujeres que hablan quechua en los Andes del sur del Perú. Históricamente, las esterilizaciones masivas de personas rurales (a menudo indígenas) en la década de 1990 reflejan los riesgos reproductivos para estas mujeres (Ballón, 2014; Tamayo, 2014; Ewig, 2014). Cuando interviene el factor racial, las categorías de «riesgo» pueden amplificarse en la percepción del Estado, en especial cuando un determinado grupo puede considerarse desviado reproductivamente (Bridges, 2011, 2017). Las siguientes secciones presentarán y analizarán la ciudadanía materna pobre de las mujeres quechuas a través de un enfoque intersectorial de la atención médica y el bienestar.

\section{METODOLOGÍA}

Esta investigación se basa en el trabajo de campo etnográfico antropológico de un año en el departamento andino del sur peruano de Ayacucho, llevado a cabo como parte de los requisitos para un doctorado en antropología médica en el University College de Londres. 
Se eligieron tres instituciones de salud pertenecientes al Minsa en la provincia sureña de Vilcashuamán: un centro de salud grande, un centro de salud pequeño y un puesto de salud satélite. Esta selección permitió obtener una amplia gama de conocimientos en diversos niveles en la cadena Minsa, aunque sus ubicaciones exactas permanecerán anónimas. Esta provincia fue seleccionada a pedido del Minsa-Ayacucho, y también debido a la presencia de una gran población empobrecida de habla quechua en esta región.

Todos los participantes eran bilingües en español y quechua, aunque en el censo de 2017 — que incluía una pregunta sobre el origen étnico- se autoidentificaron como quechuas (Chirapaq, 2017), por lo que en adelante se los denominará como tales.

La observación participante se realizó tanto en las instituciones de salud como en las comunidades locales, donde el autor vivió durante el trabajo de campo. Dentro de las instituciones de salud, la observación se realizó principalmente en las áreas de espera, y cuando se otorgó el permiso, en las consultas con los pacientes. Además, asistieron a otras reuniones y eventos relacionados con Minsa y Juntos, así como la asimilación general del autor en la mentira local durante el trabajo de campo. El investigador peruano Ramírez Hita destaca cómo la recopilación de datos etnográficos a través de la observación participante puede ser particularmente útil para complementar la investigación epidemiológica (2009). En su investigación sobre la salud quechua, ha argumentado que los datos epidemiológicos y las técnicas cuantitativas estrictas no representan las realidades locales $(2013,2009)$, por lo que defiende un enfoque etnográfico para que se presente la «imagen completa».

En total se realizaron cien entrevistas semiestructuradas: setenta con mujeres usuarias de servicios de planificación familiar y miembros de Juntos, diez parejas / pacientes varones y veinte trabajadores de la salud, así como entrevistas informales adicionales con las partes y organizaciones interesadas. Las mujeres tenían entre 18 y 49 años, y los trabajadores de salud entrevistados eran obstetras, enfermeras, técnicos y médicos. Las entrevistas se grabaron con el consentimiento del participante y luego se transcribieron. Los datos se codificaron posteriormente para su análisis.

El permiso ético fue otorgado por Minsa-Ayacucho-Diresa y University College London. Todos los nombres y ubicaciones son anónimos para mantener la confidencialidad. 


\section{ASISTENCIA SOCIAL DEL ESTADO: CUIDANDO A LOS NIÑOS}

El Estado peruano ofrece programas de asistencia social a familias de bajos ingresos, además de la atención médica gratuita que se brinda a través del sistema SIS, y la más importante de estas se dirige explícitamente al mejoramiento de los niños. De hecho, los programas de desarrollo y salud en el Perú se han enfocado durante mucho tiempo en la relación madre-bebé, pues consideran que esto requiere la mayor atención, inversión y atención. En relación con la salud pública, las mujeres y los niños han sido los clientes «primarios» debido a una historia que consideraba «el moldeado de las madres como elemento central para la construcción y mejora de la nación» (Ewig, 2010, p. 17).

Los dos programas de asistencia social con mayor visibilidad y cobertura en Vilcashuamán son Juntos, un programa de transferencia de efectivo condicional del Ministerio de Desarrollo e Inclusión Social (Midis), y CunaMás, un programa de educación y acompañamiento para padres. Ambas organizaciones tratan explícitamente la relación madre-hijo, aunque son ligeramente diferentes. Juntos proporciona una suma mensual de $\mathrm{S} / 100$ para cubrir los costos relacionados con el niño, con la condición de que los padres se aseguren de que asista a la escuela y a los controles de salud, y que la madre misma también se comprometa a contactar regularmente con su organizador de Juntos reuniones mensuales. En Vilcashuamán, los miembros de Juntos también tuvieron que asistir a días de pruebas de frotis masivos en el centro de salud regional y participar en eventos del Minsa, y algunos programas de Juntos exigen explícitamente que sus miembros utilicen la planificación familiar (Molyneux y Thomson, 2011) - aunque esto no es un evento nacional - como condición oficial del recibo de pago. De cualquier manera, el programa centrado en el niño implica el compromiso de la madre padre más allá de los factores que se relacionan directamente con su hijo receptor de la asistencia social. Los representantes de CunaMás visitan a las nuevas madres en su hogar para controlar su interacción entre padres e hijos, aunque donde CunaMás tiene un centro establecido, el programa también funciona como una forma de educación infantil y educación para padres durante el día. La capital de Vilcashuamán posee tal centro: una belleza brillante, blanca y construida con ladrillos, ubicada entre los desmoronados exteriores de adobe de las viviendas locales. Este programa también está dirigido explícitamente a la mejora de los niños a través del asesoramiento y la educación de la madre, quien se considera la principal proveedora de cuidados. 
A diferencia de estos dos programas, la planificación familiar bajo el SIS no se supone explícitamente como un servicio estatal de asistencia social dirigido a la mejora de los niños pobres, sino como un servicio médico utilizado exclusivamente por adultos o adolescentes que tienen relaciones sexuales, y es administrado por un ministerio completamente diferente de los demás, al menos a primera vista. Sin embargo, al igual que el programa Juntos - que usa el aspecto condicional de la transferencia de efectivo para influir en los comportamientos de las mujeres más allá del mandato del programa—, el servicio de planificación familiar ejerce un poder similar en reversa: un servicio dirigido y utilizado exclusivamente por adultos enmarcado como uno cuyo objetivo es beneficiar específicamente a los niños. De hecho, el centro de salud y el programa Juntos (más que CunaMás) están íntima y públicamente relacionados. Los eventos de prueba de frotis condicional son un ejemplo de cómo los trabajadores de la salud utilizan el atrapamiento de las mujeres dentro del mandato de Juntos para cumplir sus propios objetivos, en colaboración con los coordinadores de Juntos. Sin embargo, no permiten que las mujeres participen únicamente en eventos privados de Juntos en el puesto de salud, sino que deben apoyar públicamente al centro de salud y mostrarse como receptoras de ayuda del gobierno para garantizar la continuación del pago. La participación obligatoria en un desfile de «maternidad» del Minsa presenta el mejor ejemplo de esto:

Mayo de 2018. Una pancarta colgada en la puerta principal del centro de salud anuncia con orgullo la «Semana de la Maternidad Saludable y Segura». Los materiales oficiales de la campaña incluyen las palabras adicionales «y voluntaria» al final de ese eslogan, pero ese detalle de agencia y libertad reproductiva no aparece en los materiales promocionales aquí en Vilcashuamán. Hemos pasado la mañana inflando suficientes globos para entregar a todos en la procesión de la mañana que está programada para filtrarse por la ciudad y llegar a la mitad de la plaza de armas justo a tiempo para el almuerzo, y la presencia garantizada de la mejor posible audiencia.

Me empujan manojos de globos a mis manos y me doblan los codos mientras me arrastran hacia un grupo expansivo de mujeres locales que se apiñan en el patio del centro de salud. Cuando empiezo a pasarlos, noto que las manos en las que estoy distribuyendo triajes con globos son familiares: todas estas mujeres las he visto antes en las reuniones de Juntos. Aprendí que su participación en este desfile es condicional, además de la otra lista de actividades para las que aparentemente están de guardia, si quieren recibir la suma mensual de asistencia social. 
Me desplazan al frente de la procesión para acompañar a los trabajadores de la salud y ser uno de los más altos allí (como de costumbre). Se espera que levante la pancarta del centro de salud.

Comenzamos a marchar, cantando a medida que avanzamos: «iSi estas embarazada, acude a tus controles!», «¡Wawita sana, mamita feliz!»; «¡El Perú se desarrolla y avanza!», y finalmente, con uno de los trabajadores de la salud a la cabeza, «¡No al embarazo no deseado!». «iNO!». La procesión responde con entusiasmo.

Las madres de Juntos han estado detrás de nosotros, agitando sus globos y entusiastamente uniéndose al canto. Algunos han traído niños pequeños, tal vez porque no tenían a nadie con quien dejarlos en casa. Cuando el desfile se detiene en la plaza, noto que la mayoría de las mujeres se escabullen, y es probable que vuelvan a cualquier actividad que hayan tenido que abandonar para apoyar el capricho del centro de salud.

¿Estaban allí solo para inventar números o para actuar como una advertencia visual para el resto de la comunidad de cuáles son las consecuencias humillantes del «embarazo incontrolable» y la pobreza? ¿O el centro de salud realmente confió en sus números y apoyo para ayudar a promover el cuidado de la salud y embarazos seguros para otras mujeres en la aldea?

De cualquier forma que se mire, las madres de Juntos tuvieron pocas opciones para rechazar la demanda del centro de salud de que participaran en su autopromoción. Teniendo en cuenta el tema del desfile, se podría argumentar que su presencia se utilizó para actuar como un cuento de advertencia para el resto de la ciudad que salió a vigilarnos: la maternidad y la pobreza (descontroladas) resultarán en su humillación para su comunidad grande. Además, demuestra la confusión de las líneas entre los programas estatales de desarrollo (Juntos es del Midis) y las actividades del Minsa, en la maternidad en que se encuentran. El problema aquí es que la planificación familiar no necesariamente tiene que incluir a los niños directamente, a menos que hablemos de evitar su existencia. De hecho, no es un servicio que los niños puedan usar en absoluto: los anticonceptivos son estrictamente para adultos. Además, es un servicio que tiene el potencial de contribuir al desarrollo personal de proyectos de vida adulta fuera de la familia, ya que, en teoría, la anticoncepción puede respaldar el aplazamiento o el espaciamiento de los hijos donde las familias no deseadas podrían obstaculizar objetivos personales. Sin embargo, tomando una hoja del libro de sus compañeros de cama del programa de desarrollo, podría decirse que el discurso y la ejecución del programa de PPFF coloca a los niños como su beneficiario central, perdiendo 
muchas oportunidades de considerar el potencial empoderamiento de los usuarios adultos. Están representados como conductos para la (re)producción de niños peruanos exitosos y apropiados (y limitados).

Para utilizar el centro de salud más grande de la capital provincial como un caso de estudio, la geografía espacial de las salas de consulta y tratamiento es más reveladora en cuanto a los destinatarios de cada servicio. El centro de salud elegido consta de tres edificios principales y tres construcciones auxiliares más pequeñas dentro de un muro vallado, protegido por una imponente puerta de hierro que siempre está abierta. Los edificios están dispuestos alrededor de una plaza central donde los pacientes a menudo se sientan y esperan cuando hace buen tiempo; en esto, refleja la configuración típica de la mayoría de las aldeas, pueblos y ciudades que se encuentran en todo el Perú.

Aquí son de interés los dos edificios principales (el tercero se usa principalmente para eventos del personal y oficinas privadas). Uno es el edificio de «maternidad». Contiene todas las salas de consulta y el equipo que uno podría asociar con los servicios generales de maternidad y de mujeres: una sala de partos, equipo de prueba de frotis, máquina de ecografía, etc. El otro edificio principal alberga medicina más general, pero podría verse cómodamente como el edificio para niños. Aquí es donde se pueden encontrar las salas de consulta de «crecimiento y desarrollo» y «estimulación temprana», y donde se encuentra una modesta área de juegos suaves para niños.

Invito al lector a adivinar dónde se encuentra la sala de consulta de planificación familiar: ¿en el edificio de servicios para mujeres, o enclavado entre los servicios para niños? El preámbulo de este análisis revela la respuesta: está ubicado entre los servicios para niños. Por supuesto, siempre se podría argumentar que las mujeres que usan el edificio de maternidad ya pueden estar embarazadas, por lo que no necesitan acceso inmediato a la anticoncepción, sin embargo, se debe afirmar que la mayoría de los obstetras (y todos sus archivos y equipos de pacientes) están ubicados en el edificio de maternidad [relevante], no entre las enfermeras y médicos que atendieron a los niños, por lo que incluso las mujeres no embarazadas se dirigirían lógicamente al edificio de maternidades para la anticoncepción, ya que son los obstetras quienes se encargan de este servicio. En cambio, se podría sugerir que la geografía espacial del centro de salud subraya algo más fundamental; la planificación familiar está incluida en los servicios para niños porque se considera un servicio para niños. En este sentido, no se pretende decir que se ofrece anticonceptivos a los niños, sino que los principales beneficiarios de la planificación familiar se interpretan como niños, y no como mujeres, hombres o parejas. 
Este discurso sobre la planificación familiar dirigido a los niños se repetía una y otra vez en la sala de consulta, y generalmente, cuando se reprendía a una mujer por «exceso» de nacimientos y se burlaba de que debía cesar para concentrarse en apoyar a la descendencia, que ella ya lo había hecho. Como se lamentaba un obstetra, cuando la madre vuelve a quedar embarazada, «los hijos sufren». Una vez que una mujer tuvo un hijo, los trabajadores de la salud elogiaron las virtudes del uso sostenido de anticonceptivos, con un enfoque particular en el implante de tres años que podría asegurar un espacio exitoso durante un período prolongado. Estaba imbuido de una mayor conveniencia a través de las representaciones de obstetras de su rareza. Por ejemplo, en una de las publicaciones de salud más pequeñas, observé una consulta en la cual la obstetra agitó el método en caja frente a una paciente, diciéndole: «Estos son caros, no tenemos muchos, aprovecha», presumiblemente porque creía que el método se volvería instantáneamente más atractivo para esta mujer a través de su supuesta exclusividad y valor financiero. Tal enfrentamiento resalta la suposición del obstetra de que las mujeres más pobres pueden ser seducidas por cosas costosas, que están interesadas (discutido más adelante). Este paciente dijo que no, pero otros aceptaron voluntariamente el método después de una conferencia sobre limitación de niños y cómo el exceso de niños dañaría el futuro de los demás a través del consumo de recursos.

De hecho, cuando una mujer más pobre se convierte en madre, se ve involucrada en los variados programas de asistencia social del estado que, de formas variadas pero interrelacionadas, comienzan a ignorarla como individuo y a centrarse únicamente en su valor como vehículo a través del cual los niños pueden ser «adecuadamente» elevados. Esto se ve como una imposibilidad dentro de las familias más grandes que se supone que tienen las mujeres más pobres, y por lo tanto, la limitación de niños surge como un discurso central en el centro de salud y más allá. Casi todos los trabajadores de la salud lamentaron que la fertilidad descontrolada y la falta de recursos para apoyar a esos niños fueran un tema clave que debía superarse. Por lo tanto, limitar los nacimientos se promueve no como un objetivo para reducir los problemas de una mujer y o mejorar su forma de vida, sino como un deber materno para con los hijos vivos.

En su etnografía sobre la amniocentesis en los Estados Unidos, Rapp (1999) se refiere a la difícil decisión que toman las mujeres para interrumpir el embarazo de niños discapacitados como una especie de «altruismo materno» (pp. 249-250). Cuando una madre descubre que su feto esperado tiene una discapacidad, se le presenta la opción de interrumpir el embarazo como una forma de «altruismo» para sus otros hijos y para ella misma. La decisión de mantener a un niño discapacitado, 
en estos casos, podría haber significado que los miembros de la familia existentes sufrieran, por lo que la mujer tiene que tomar una decisión difícil. Se podría argumentar que, en Vilcashuamán, las mujeres también deben mostrar «altruismo materno» al limitar el nacimiento de hijos que de otro modo hubieran querido, para proteger a los miembros de la familia existente del sufrimiento.

Aunque el discurso no rodea la discapacidad física, se centra en un tipo diferente de discapacidad: los recursos. Los niños adicionales pueden agotar los recursos disponibles para aquellos que ya están vivos, dice la narración, y por lo tanto, se espera que la mujer muestre altruismo materno, limitando sus nacimientos. La forma de lograr esto — sugiere el puesto de salud - es a través del uso de anticonceptivos biomédicos. Por lo tanto, se alienta a las mujeres pobres a utilizar los servicios de PPFF y la anticoncepción como una forma «altruista» de atender a sus hijos existentes, en lugar de considerar las motivaciones personales para evitar el embarazo, por ejemplo. Esta es una narración complicada que se extiende más allá de las preocupaciones estatales «inocentes» por el futuro de los niños. Lo que tienen en común los diferentes programas (SIS-FP, Juntos y CunaMás), además de estar dirigidos a la mejora de los niños que usan a la madre como vehículo, es que todos están dirigidos específicamente a madres pobres.

Bridges analiza cómo funciona una «construcción moral de la pobreza» para sugerir que las personas pobres carecen moralmente de alguna manera, o son pobres debido a la falta de ética laboral, pereza o sexualidad sin restricciones. Las madres pobres preocupan especialmente a los gobiernos, ya que estas mujeres, «según las narrativas culturales dominantes, tomaron la decisión inmoral de permitir que su pobreza se cruzara con su maternidad. El peligro es que podrían tomar decisiones inmorales con respecto a los niños a su cargo» $(2017$, p. 80). Simplemente por ser pobre, y al recibir la asistencia social, como los fondos de Juntos o los anticonceptivos gratuitos bajo el SIS, estas mujeres pueden considerarse automáticamente como más propensas a descuidar a sus hijos, de ahí la insistencia del enfoque del programa gubernamental en la relación madre-hijo, y el mantra de la salud de la limitación infantil.

Bridges sugiere, además, que estas mujeres no disfrutan de la ciudadanía plena debido a su situación: «La convicción de que las deficiencias morales causan pobreza sirve para justificar la privación de las madres pobres... su ciudadanía es parcial. Son de segunda clase o semiciudadanos» (p. 55). Podría decirse que su estatus de ciudadanía se vuelve dependiente de su participación «buena»en las actividades de asistencia social: asistir a las reuniones correctas, unirse al desfile y usar anticonceptivos. Gillespie llama a esto «ciudadanía potencial». 
Con base en su investigación sobre Juntos en Ayacucho, concluye que «las madres son abordadas no como personas que se supone que son ciudadanas, sino como ciudadanas potenciales que tienen que promulgarlas primero. La inclusión social no parece estar basada en la suposición de que ser ciudadano es un estado automáticamente inclusivo, sino que es un proceso cargado de valores que requiere respuesta y acción individual» (2016, p. 200).

La ciudadanía plena es la zanahoria que se encuentra fuera del alcance de las madres pobres, siempre y cuando cumplan con las instrucciones de su coordinador de Juntos y los obstetras del Minsa. Por lo tanto, se podría argumentar que, si bien el discurso puede diferir para las mujeres más ricas (como concluye Bridges, por ejemplo), el delito menor de ser madre mientras es pobre es motivo suficiente para que las mujeres en Vilcashuamán experimenten la revocación de su ciudadanía plena, solo para ganarse la vida, y volver si se desempeñan satisfactoriamente como madres. Lejos de ser un comentario sobre todas las maternidades, ser una madre pobre en la zona rural de Ayacucho es perder el valor a los ojos del Estado: ese valor solo se puede canjear si se pastorean a los hijos existentes a una meta predeterminada.

\section{UNA FUTURA GENERACIÓN DE «PROFESIONALES»}

María Eugenia acababa de dar a luz a su sexto hijo y se inclinó sobre el certificado de nacimiento recién impreso, sintiendo en silencio las palabras en español en su boca mientras seguía las oraciones lentamente con el dedo. Me senté para ofrecerle mi ayuda con tacto, notando que podría ser analfabeta y entablar una conversación informal mientras esperaba que el equipo médico entrara y la diera de alta del centro. Su recién nacido estaba envuelto en una manta y durmiendo profundamente; sus otros hijos se fueron a su casa en su pueblo con el mayor, un niño de trece años, para cuidarlos. Le pregunté a María si estaba pensando en tener más hijos en el futuro, una pregunta con la que se rio. «No», dijo ella, «el costo es demasiado alto»:

RI: ¿Qué costos hay?

María: ¡Oh, la educación!

RI: Pero la educación es gratis, ¿o no?

María: Sí, pero te cuesta el uniforme, sus cuadernos, mandarlos a estudiar...

RI: ¿Y qué quisieras que estudien? 
María: No sé, que sean profesionales.

RI: ¿Como doctores o ingenieros?

María: No sé, no importa, lo que ellos quisieran estudiar, pero que sean profesionales, pues.

Convertirse en un «profesional» es un deseo frecuentemente citado para los hijos, incluso si las personas rara vez pudieran delinear una profesión en particular que quisieran que sus hijos sigan. La profesión en sí no es necesariamente importante, pero sí el aumento asociado en el Estado y el cambio de estilo de vida. Este es un objetivo bastante común para los jóvenes en todo el Perú, como demuestra Crivello (2011) en un estudio que abarca diferentes regiones del país y que ofrecen variaciones sobre esta misma idea. La profesionalización está estrechamente relacionada tanto con la educación como con la migración, factores que supuestamente pueden ofrecer una «vía de salida de la pobreza» (p. 1) para las personas desfavorecidas: la introducción de una «educación intercultural» en el Perú, que se suponía un programa nacional, pero que se enfocaba principalmente en áreas rurales e indígenas (García, 2010). En su estudio sobre Ayacucho, Cavero Carrasco (2008) subraya cómo esta educación, una supuesta vía de salida de la pobreza y el factor migratorio, sigue siendo más teórica y rara vez llega a los pueblos indígenas en un sentido práctico. Por lo tanto, debido a la falta de instalaciones de educación superior en las aldeas rurales, la obtención de estudios adicionales debe realizarse necesariamente en los centros urbanos. Para la gente de Vilcashuamán, esto significa Huamanga (capital departamental de Ayacucho) o Lima.

Sin embargo, el concepto de convertirse en profesional no solo se asocia con mayores ganancias. La naturaleza misma de la profesionalización significa migración, y eso es casi siempre de los centros rurales a los urbanos (Crivello, 2011). Esto también implica un cambio del trabajo agrícola al empleo urbano y un estilo de vida completamente diferente del de sus predecesores. Además, y lo más importante, la migración rural-urbana puede sugerir una migración de identidad personal y racial (Ewig, 2010, p. 15): de campesino a habitante de la ciudad, del quechua al castellano, de indio a mestizo o blanco (Weismantel, 1992). Convertirse en un profesional, por lo tanto, puede resultar en la desintegración de un campesino, con el descarte de todos los conceptos asociados inherentes a la transformación. Como también descubrieron Tubino y Zariquiey (2007), los peruanos indígenas en la Amazonía descubrieron que sus costumbres y su corte cambiaron dramáticamente como resultado de la interacción con lo urbano, donde las complicadas relaciones de poder y discriminación entraron en juego. 
Aquí, una experiencia similar podría estar teniendo lugar con los quechuas de Vilcashuamán, lo que sugiere que una migración física a través del espacio podría resultar en una migración teórica a través de la raza. El centro de salud felizmente promueve esta idea.

En las paredes de las publicaciones de salud se muestra un póster de suplementos nutricionales diseñados para reducir la anemia en los niños (ver imagen 1). Inocentemente, el póster alienta a los padres a alimentar a sus hijos con muchos alimentos apropiados, complementados con el polvo biomédico, durante seis meses, para curar la anemia de un hijo. Una burbuja de diálogo dice: «Sin anemia tus wawitas serán grandes profesionales», con una flecha que dirige la mirada del público a fotografías de los «grandes profesionales» sugeridos: una niña con una chaqueta médica y un hombre con un casco amarillo (presumimos ingeniero). Lo interesante es la yuxtaposición de dichos profesionales con los «no profesionales». Separados por las bolsitas rosadas de suplemento nutricional están los profesionales y los niños «antes»: una niña usa dos trenzas largas y un bombín, que pueden identificarse como ropa / peinado indígena quechua. Junto a ella hay un niño pequeño que, aunque usa un vestido no indígena, tiene las mejillas claramente quemadas por el sol, lo que es una característica distintiva de los niños de las tierras altas (y, por lo tanto, potencialmente indígenas) debido a las condiciones climáticas extremas del sol directo y los fuertes vientos que crean la grieta, mejillas enrojecidas y rojizas distintivas de las personas que viven en las montañas. En pocas palabras, los niños «antes» pueden identificarse como más indígenas.

Sin embargo, cuando uno recurre a los «profesionales», observamos una piel más clara, quizás incluso mejorada a través de una mayor exposición a la luz en las fotografías. El cabello de la mujer es liso y sin trenzar, el hombre tiene una tez suave. En este póster, la sugerencia es que alimentar a sus hijos con suplementos nutricionales puede convertirlos en profesionales. En esta narrativa, los profesionales son blancos, mientras que los no profesionales son niños indígenas anémicos. Aunque un suplemento nutricional no puede cambiar el origen étnico de una persona, la capacidad de la biomedicina para «blanquear» a una persona ha sido reconocida en la literatura (en relación con la salud reproductiva, ver Roberts, 2012a, 2012b). Sin embargo, también se puede suponer que, además de la biomedicina, «convertirse en un profesional» también puede blanquear a una persona. Por lo tanto, cuando las personas hablan de querer que sus hijos se conviertan en profesionales, también pueden estar señalando un deseo de convertirse, si no étnicamente blancos necesariamente, al menos en menos indígenas. 
García discute la relación de larga data del Perú con «el problema indio» y la educación, un mecanismo de profesionalización, y destaca cómo todos los proyectos de creación de naciones que abordan dicho «problema» se han centrado notablemente en el idioma y la educación de los pueblos indígenas. Además, sugiere que la educación es «uno de los principales mecanismos a través del cual los indios se transformarían en ciudadanos» (2015, p. 63) (énfasis agregado). Como el discurso de profesionalización que prevalece en los puestos de salud insinúa la necesidad de educación (y la migración que lo acompaña), se puede sugerir que esto también contribuye a un discurso generalizado sobre ciudadanía e «indigeneidad». Después de García, si se necesita educación para transformar a una persona indígena en un ciudadano, se podría suponer que, antes de esta intervención, no pueden disfrutar de la ciudadanía plena. Por lo tanto, los hijos de las mujeres rurales pueden obtener esta ciudadanía a través de la profesionalización y la posterior transformación, pero sus madres nunca podrán hacerlo, analfabetas y sin educación, como muchas de ellas.

Desde la perspectiva de los centros de salud, el estímulo a la profesionalización también puede sugerir una percepción de que el estilo de vida actual de las personas no es lo suficientemente bueno. Como un obstetra le dijo a un paciente durante una consulta:

¿Acaso no quisieras que tus hijos te superen? Mírame, mi mamá trabaja en el mercado y mi papá en la chacra, pero aquí estoy, un profesional. Fui a la universidad y ahora logré ser mejor que mis papás.

Querer que los hijos de uno logren algo no es necesariamente un problema. Sin embargo, la sugerencia de que el estilo de vida actual no es lo suficientemente bueno puede tener más implicaciones más allá de las de la superficie. Por ejemplo, la narrativa subyacente sugiere que la agricultura y el pastoreo de animales (mercados y chacras) son formas de vida indeseables. Sin embargo, trabajar la tierra es mucho más que un simple trabajo para muchas personas, ya que el entorno natural sigue teniendo un significado espiritual en Ayacucho y otras partes de los Andes. Es una forma de vida laboral, pero también una cosmología. La sugerencia de que la próxima generación necesita abandonar este trabajo no solo aborda el estilo de vida y el salario, sino que también puede leerse para sugerir un abandono de las creencias y la relación con el mundo natural, un elemento central de las realidades indígenas locales.

Finalmente, el enfoque en la profesionalización pasa por alto una realidad deslumbrante; incluso si una persona indígena migrara y se educara para conver- 
tirse en un «profesional», las barreras estructurales existentes en el Perú pueden prohibirle que alcance realmente este objetivo que el discurso dice que debería.

Sobre esto, Goldberg avanza la teoría del «posracialismo», una afirmación sobre el estado de la sociedad en la que vivimos, o deberíamos vivir, «fuera de la referencia racial debilitante [...] supone que el esfuerzo, la energía y la capacidad inherente determinarán a los individuos "perspectivas de vida"» $(2015$, p. 2). Goldberg no sugiere que la «raza» haya terminado, sino que las expresiones de racismo y las formas de pensar sobre la raza están experimentando un proceso de cambio. Ahora se considera que depende de la voluntad individual, las opciones de vida, las aspiraciones personales y el trabajo duro que las personas existan de ciertas maneras en el mundo, no debido a su «raza» y acompañando posibles discriminaciones y prejuicios, como dice el argumento.

Sin embargo, como la «raza», tal como la conocemos, se vuelve cada vez más invisible en el discurso público, se alienta al racismo a vivir «sin marcar, incluso sin ser reconocido, potencialmente para siempre» (p. 6). Para volver al cartel de nutrición, entonces, como un ejemplo, aquí no vemos mención de raza, no entra en la discusión. Estamos hablando de la desnutrición, que es producto de la pobreza y la falta de acceso a los recursos. Si los padres toman la sabia decisión de dar suplementos a sus hijos, estos se convertirán en «grandes profesionales» y saldrán de la pobreza, sugiere. Sin embargo, es evidente que los profesionales del cartel son étnicamente más blancos que el público objetivo indígena del cartel, por lo que se aborda la raza. Como sugiere Goldberg, se ha vuelto más sutil, sin marcas y, por lo tanto, más penetrante.

Entonces uno podría plantear la pregunta: ¿qué pasa con la realidad de esta profesionalización?

Maribel, hija de un miembro de Juntos, había regresado para visitar su pueblo natal de Huamanga, donde ahora vivía con su tía. Después de que sus padres tuvieron problemas para comprar todo el equipo escolar necesario y las tarifas de transporte, finalmente pudieron enviar a su hija a vivir a la ciudad y estudiar administración en una institución educativa. Regresó durante las vacaciones para visitar la casa de su familia, donde la conocí, y mientras comíamos pescado frito me contó sobre su vida en la ciudad. La casa de sus padres era humilde, un edificio de adobe con techo de hojalata, agua corriente y algo de electricidad, construido hábilmente en la ladera de una colina traicionera para escalar cuando la temporada de lluvias convierte la tierra en fango, pero típica de las aldeas. Aparentemente, Maribel había escapado de todo esto por haber sido enviada lejos para convertirse en una «profesional» en la gran ciudad. Hablaban intermitentemente en quechua y 
español, alternando entre los dos cuando sentían que me estaba perdiendo con los quechuas. Como a menudo pasaba por Huamanga, Maribel mencionó que debía visitarla. Ahora estaba trabajando en una farmacia y podía preparar un almuerzo en su casa cuando yo llegara. Acepté su invitación.

La casa que Maribel compartía con su tía en Huamanga no era un apartamento de gran altura con piso de madera con ascensor y balcón en el centro de la ciudad, como podría sugerir la vida en la ciudad, sino una estructura de adobe en una colina polvorienta muy lejos de la plaza de armas. Los vecinos eran en su mayoría campesinos de todo el departamento, me dijo. "Allinllachu», se saludaron en quechua al pasar por la calle. Su lugar de trabajo era una pequeña farmacia de propiedad independiente que almacenaba algunos artículos alimenticios y medicinas, aparentemente para compensar las ganancias. No era una de los InkaFarma o MiFarma franquiciados, con sus distintivos letreros de tiendas de color amarillo neón y naranja y sus uniformes llamativos.

De hecho, esta vida no era lo que esperaba encontrar cuando visité a una vilquina que había logrado el sueño de «profesionalización». La existencia actual de Maribel era bastante similar a la que vivían sus padres en el pueblo. No se había convertido en ingeniera o doctora como prometía el cartel, tal vez por carecer del capital social y financiero para perseguir este sueño en particular incluso si lo hubiera deseado. Su situación de vida no era muy diferente de la que le quedaba, con una excepción: había dejado la aldea y ahora podía decir que vivía en la ciudad, con todos los cambios asociados que esta migración conllevaba. Si las realidades de la profesionalización no son materiales, entonces deben ser otra cosa. Maribel ya no era una campesina destinada a una vida agrícola, sino una chica de ciudad «profesional». No importaba que casi nada hubiera cambiado materialmente. Al seguir el discurso de la profesionalización, podría decirse que ahora se llama a sí misma ciudadana del Perú de una manera que sus padres empobrecidos, que trabajan en las chacras y reciben asistencia social del Estado, no pueden.

Barrig (2001) describe este mismo proceso de «desindigenización» que les ocurre a las mujeres andinas cuando se mudan a Lima para trabajar como empleadas en las casas de las peruanas blancas:

Las empleadas domésticas, migrantes andinas a Lima, habían empezado ellas mismas su camino hacia la «desindigenizacion». El solo hecho de mudarse a una ciudad grande y vivir en una casa desde la cual habrían de absorber patrones de la vida urbana, incluso en un trabajo considerado denigrante, implicaba un giro social de ascenso respecto de su comunidad de origen, y de huida de las nociones predominantes de inferioridad de lo andino (2001, p. 59). 
Aunque a las mujeres indígenas se les dan espacios de vida pequeños y estrechos, pueden enfrentar malas condiciones de trabajo y están mal pagadas (2001, p. 45), el mismo acto de emigrar a una ciudad comienza un proceso de blanqueamiento, como señala Barrig, y posiblemente como Maribel también buscó. No se trata necesariamente de aumentar los salarios o incluso las condiciones de vida: se trata de una transformación racial subjetiva que se puede buscar a través de «huirse» de la comunidad andina.

\section{PRIORIZACIÓN FINANCIERA}

Siguiendo la teoría del posracialismo de Goldberg y su enfoque en el logro individual en lugar de las desigualdades estructurales, uno ya puede haber notado la asociación implícita con el neoliberalismo (como Goldberg realmente lo hace). Bajo el neoliberalismo, «cada individuo es responsable de sus propias acciones y bienestar» (Harvey, 2007, p. 65), como tal «el posracial individualiza la responsabilidad» (Goldberg, 2015, p. 62). Es importante destacar que el neoliberalismo requiere que el individuo interactúe con los mercados de una manera inteligente: «El éxito o el fracaso individual [en el mercado] se interpretan en términos de virtud empresarial o fallas personales (como no invertir lo suficiente en el propio capital humano a través de la educación) en lugar de atribuirse a cualquier propiedad sistémica (como las exclusiones de clase por lo general atribuido al capitalismo)» (Harvey, 2007, pp. 65-66).

El neoliberalismo presupone - de hecho exige — una fluidez financiera y de mercado que las madres indígenas pueden nunca haber experimentado antes de su incorporación al redil de la planificación de bienestar familiar y el discurso del centro de salud.

Las finanzas y la planificación familiar se mencionan una y otra vez en los centros de salud. Todos los trabajadores de salud mencionaron consideraciones financieras como una razón para limitar la maternidad en sus entrevistas, y los pacientes estuvieron constantemente expuestos a dar conferencias sobre sus finanzas. En una reunión de Juntos, donde el efectivo está a la orden del día, esto puede esperarse. Sin embargo, en asuntos de planificación familiar y parto, donde los trabajadores de la salud están encargados de atender el bienestar de la paciente y no su cuenta bancaria, la existencia de conversaciones de dinero puede ser menos relevante. De hecho, en su historia de planificación familiar en Perú, Necochea López señala explícitamente que esta suposición de priorización financiera en relación con el espaciamiento de los nacimientos debe ser cuestionada (2016, p. 11). 
Sin embargo, la relación entre los niños - y específicamente el número de niños - y las finanzas fue un tema de obsesión, tanto directamente en las consultas, como indirectamente en la sala de posparto:

Mayo de 2018. La sala de maternidad está inusualmente ocupada, con tres nuevas madres ocupadas con sus recién nacidos cuando sus hijos y parejas entran y salen de la habitación. El espacio está lleno y animado, como los archivos habituales de séquito postparto en; un médico, un obstetra, una enfermera, un nutricionista, un técnico, un trabajador de CunaMás, todos los que comienzan a revolver formularios y a hablar en voz alta y decididamente sobre el ruido.

«iAy, para tener hijos en estos días tendrías que ser millonario!», la técnica se ríe de sus colegas.

El médico responde sin querer mientras marca algunas casillas en un formulario: «Sí, cada niño le costará S/ 360 cada mes para mantener».

«Tienes que ser rico para tener hijos en estos días!», contesta otra voz.

El médico recurre a una de las madres que hasta ahora había sido excluida de esta conversación y se ha ocupado de sus propios asuntos, para preguntar: «¿Cuántos hijos tiene?».

«Seis», ella responde.

« $\mathrm{Oh}$, debes ser muy rica!», afirma, con cara de pan.

Su jersey está sucio y un poco irregular, y noto que las suelas de sus zapatos se están despegando. Ella ciertamente no se ve rica.

La enfermera interrumpe: « ¡A no! ¡No creo que pueda tener hijos! ¡El costo es demasiado!». El técnico está de acuerdo, respondiendo que con su único hijo apenas se las arregla.

«¿Cuántos tiene, doctor?», pregunta ella.

«Yo, ninguno».

Luego, el técnico le pregunta al obstetra: «¿Cuántos hijos tiene?» La respuesta es: «Dos».

«Ah, sí, eres rico entonces», bromea el doctor ante la risa de todos.

Para esta mujer, señalada frente a toda la habitación, su cama ya estaba hecha. Ella ya tenía seis hijos, una indignación para los trabajadores de la salud, por lo que fue humillada por esto. Además, los trabajadores la comparaban desfavorablemente con ellos mismos, menospreciando a ella por no tomar las mismas decisiones que habían tomado personalmente sobre el tamaño de la familia. 
Esta es la consecuencia de no seguir el mandato del centro de salud, y las críticas a menudo se dirigieron a las mujeres que ya tenían varios hijos. Otra mujer que había recibido una vergüenza similar criticó a los trabajadores de salud: «¿Quiénes son para tratarnos así? Ellos solo trabajan con la salud, ellos no pagan nada, yo pago mis cosas, que no se metan».

Aunque su indignación por el maltrato puede estar justificada, tales opiniones son efectivamente incorrectas con respecto a quién paga. Es posible que los trabajadores de salud no paguen personalmente por las mujeres, pero su empleador, el Estado, sí paga por ellas a través de su SIS. Es este bienestar el que posiblemente otorga a los trabajadores la ilusión del derecho a criticar a las mujeres que «gastan en exceso» a través de una reproducción sin restricciones. Como sugiere Bridges $(2011,2017)$, la recepción de asistencia social priva a las mujeres de la privacidad de las opciones familiares y personales, y los trabajadores de la salud reconocen esta conexión.

Por ejemplo, después de haber dado a luz a su quinto hijo, una obstetra criticó duramente a una nueva madre, llegando a sugerir que sus hijos ahora pueden enfrentar problemas de salud y muerte, debido a su fertilidad sin restricciones y su falta de capacidad para pagar los costos: «¿Quién va a pagar por todo esto? El Estado paga, por tu embarazo, tu vaso de leche, tu Juntos... y si muere tu bebé por causa de la anemia, ¿quién va a pagar? Es otra inversión por el Estado más».

Lejos de ser solo un ataque a la responsabilidad de esta mujer, en este caso la trabajadora de la salud sugirió que sus embarazos múltiples están causando una carga financiera al Estado y una «inversión» más que potencialmente no deberían estar haciendo. Un término y concepto despectivo utilizado para tales mujeres es «reinas del bienestar» (welfare queen), como sugiere Bridges (2011). El estereotipo es el de una mujer que se recuesta con innumerables hijos para cobrar los pagos de asistencia social del Estado: «Ella no tiene educación, pero está lo suficientemente informada como para hacer lucrativas sus capacidades reproductivas. Es estúpida, pero lo suficientemente inteligente como para trasladar al gobierno los costos de mantenerla» (p. 211). Ella también está racializada. La investigación de Bridges tuvo lugar en los Estados Unidos, donde la «reina del bienestar» fue retratada como negra (p. 212), una raza desfavorecida en ese contexto. En el Perú, la desventaja étnica posiblemente se aplica a las personas indígenas y no mestizas / blancas: las madres más pobres de la zona rural de Ayacucho. Sin embargo, en este contexto se expresa como mujeres interesadas: solo hacen cosas por dinero. 
Un médico creía que las mujeres no deberían recibir ningún dinero del Estado, ya que esto las hace «flojas» $\mathrm{o}$ «facilistas», queriendo la manera fácil (Gillespie, 2016, p. 76) y ha resultado en una situación en la que no harán nada a menos que se reciba el pago:

Las mujeres no deberían recibir este dinero [Juntos], necesita ser reorganizado porque solo vienen a las reuniones para obtener ese dinero, de lo contrario no vendrían. Antes la gente trabajaba en la chacra y comía de la chacra. Juntos significa que las personas solo confían en el Estado por dinero y no hacen nada por sí mismas. Cuando hay una reunión que no involucra dinero, no van. Por ejemplo, se supone que las mujeres se encontraran en la escuela la semana pasada y solo una fue. Fue porque no había dinero involucrado. Si no se les paga, no harán nada, ni siquiera por sus hijos. Son bien interesadas. Había una mujer de 37 que no acudiría a sus controles [en el centro de salud], porque dijo que ya tenía cuatro hijos y sabía lo que estaba haciendo... pero ¿cómo podría no preocuparse por su feto? ¡Bebé así! Ella dijo: «Es mi cuerpo, yo tomo mis decisiones!». No deberían seguir dándoles dinero en Juntos.

Los trabajadores de la salud pueden criticar duramente a las mujeres que dependen de los fondos del Estado para mantener a sus familias, y esto puede desarrollarse en comentarios agresivos como los citados anteriormente.

De hecho, los fondos recibidos por Juntos, aunque útiles, pueden tener una importancia mucho menor para los destinatarios que lo asumido por este médico y otros, en lugar de valorar su propio trabajo (trabajo que pueden haberse perdido para asistir al desfile obligatorio de Juntos / Minsa discutido anteriormente, por ejemplo) como mucho más importante financieramente. Por ejemplo, Gillespie descubrió que:

A las mujeres no les pareció que el pago fuera lo suficientemente insignificante como para optar por no participar en el programa, sin embargo [...] era una pequeña fracción de lo que se necesitaba para mantener a sus familias, y calificaron su propio trabajo [...] como una fuente mucho más grande de ingresos $[\ldots]$ contrario a los puntos de vista nacionales del «facilista» o campesino perezoso que vive de los programas del gobierno (2016, pp. 75-76).

Sin embargo, hay poco reconocimiento de la situación real y de la posición de los centros de salud al crearla. Por ejemplo, el médico que lamentaba que la gente solía trabajar en el campo y contentarse no parece reconocer que el discurso de profesionalización, promovido por el centro de salud, alienta activamente a las personas a abandonar las chacras y alejarse. El obstetra que atacó verbalmente a la 
nueva madre tampoco consideró que si los bebés de una mujer siguen muriendo de anemia (lo que podrían haber tenido en este caso), entonces ella podría haber priorizado el duelo o tratar de tener un nuevo bebé en lugar de preocuparse por los costos funerarios modestos, lo que de todos modos es poco probable que haya sido pagado por el gobierno. Nuevamente encontramos que el discurso del niño es limitado debido a consideraciones financieras con un valor moral adjunto, ya que es el Estado peruano el que supuestamente está asumiendo esta carga.

Sin embargo, el concepto de mujeres cada vez más interesadas se extiende más allá del centro de salud y Juntos. Después de entrevistar a miembros de la comunidad sobre sus pensamientos sobre las esterilizaciones pasadas, por ejemplo, varias personas sugirieron que las mujeres afirmaban haber sido esterilizadas solo porque el Estado ahora ofrecía una compensación financiera: las mujeres supuestamente jugaron a la víctima porque están interesadas.

Durante una entrevista con un chamán de la jungla visitante, este tema surgió una vez más. Cuando le pregunté cuáles eran las solicitudes más comunes de sus clientes, no necesitaba pensarlo dos veces: encontrar un esposo rico y dejar de trabajar. Interesadas.

Esto puede deberse a que la relación percibida entre los agricultores rurales y el dinero no sigue lo que se considera apropiado según el modelo neoliberal. La recepción de la asistencia social, o los recursos de un cónyuge niegan la necesidad de una participación individualizada en el mercado. Meltzer (2013) argumenta que, para que las mujeres que reciben asistencia social estatal en el Perú participen efectivamente en el mercado, se supone que deben seguir los ideales neoliberales de gestión de riesgos individuales y acumulación de activos, como promueve el programa Juntos, entre otros. Ella llama a esto «buena ciudadanía». La buena ciudadanía se basa en un «ciudadano fiscalmente responsable» (p. 644) que desarrolla hábitos y actitudes específicas hacia el dinero, en particular la capacidad de ahorrar y gestionar el riesgo individual (p. 647). La antítesis de esto, «mala ciudadanía», podría ser una persona financieramente analfabeta que no puede hacer proyecciones adecuadas (aquellos que tienen más hijos sin considerar las finanzas, por ejemplo), ni acumular activos o ahorrar efectivamente (aquellos que viven de la mano boca a boca, por ejemplo, al igual que muchas familias rurales pobres). Además, Meltzer señala que existe una suposición subyacente de que la «buena ciudadanía» se define por la capacidad [...] de poder mitigar de manera independiente los riesgos sociales y económicos» (p. 646), es decir, no depender del gobierno para rescatar a uno de la crisis financiera. Como la gestión exitosa de las finanzas excluye la «buena ciudadanía», las mujeres / madres 
que no planifican en familia y, por lo tanto, no atienden a la gestión financiera, reciben la «mala ciudadanía». Este tipo de ciudadanía también está racializada. Como Meltzer argumenta:

Los discursos de «buena ciudadanía» también están codificados racialmente, reconfigurando narrativas más antiguas en las que la «aptitud» ciudadana está entrelazada con las jerarquías sociales racializadas [...] Aunque las poblaciones indígenas no son explícitamente objetivo en el programa contemporáneo de transferencia de efectivo o ahorro, sin embargo, continúan constituyendo el mayoría significativa de las poblaciones extremadamente pobres en el Perú, y por lo tanto constituyen la mayoría de los destinatarios específicos [...] la pobreza y la falta de ingresos no se conciben únicamente como condiciones socioeconómicas que podrían afectar a cualquier persona en la sociedad, sino que de alguna manera están enraizadas en un atraso persistente (2013, pp. 649-650).

Por lo tanto, a las madres más pobres que también son indígenas quechuas, como las de Vilcashuamán, nuevamente se les condiciona su condición de ciudadanía plena y deseable, aquí en el «buen» manejo neoliberal de las finanzas, pero también con la condición de que desarrollen ciertos tipos de comportamientos reproductivos y pastorear a los niños existentes a una vida de profesionalización, una vida lejos de las prácticas agrícolas e indígenas de sus padres.

Entonces, las madres empobrecidas y «en riesgo» son consideradas moralmente irresponsables por quedar embarazadas mientras son pobres (Bridges, 2011). Después de esto, las mujeres son criticadas por no tener dinero y tener hijos. Sin embargo, si buscan y obtienen finanzas del Estado, son criticadas por hacerlo. Se supone que las mujeres deben alejar a sus hijos de los estilos de vida agrícolas para que puedan convertirse en «profesionales» en la ciudad; sin embargo, son criticadas por no seguir adheridos al estilo de vida agrícola «más saludable» del pasado. En resumen, no pueden ganar, ya que este discurso las coloca en una situación atrapante. La ciudadanía plena para las madres indígenas pobres es, desde este punto de vista, un objetivo inalcanzable. Sus hijos pueden obtener esto, pero solo a través de la eliminación calculada de una identidad compartida con sus padres y el cambio a una nueva.

\section{CONCLUSIÓN}

A pesar del discurso generalizado, las finanzas solo necesitan ser una parte de la historia, incluso cuando se discute el deseo de «profesionalización» y los 
mecanismos para lograrlo. Por ejemplo, como parte de las reparaciones otorgadas a las víctimas de la violencia, los hijos y nietos de los afectados tienen acceso a fondos y becas para ayudarlos a asistir a la educación superior (La República, 2016). Esta es una forma en que las personas pueden acceder a la educación sin el alto precio tan lamentado por el centro de salud. De hecho, si las madres fueran víctimas de la violencia (como lo fueron muchas personas en Ayacucho), entonces podrían pasar estas reparaciones a sus hijos si así lo desearan. Los trabajadores de la salud no mencionan esto en absoluto, pero la gente sí lo sabe. Un padre-paciente me presentó por primera vez este plan, por ejemplo, quien eligió informarme que su hija, una niña de cuatro años que tenía en sus brazos, estudiaría en Lima cuando fuera mayor, usando este mismo esquema. Uno puede preguntarse por qué los trabajadores de la salud eran reacios a mencionar esto como una alternativa.

Como otra posible solución, Leinaweaver (2009) escribe que, en Ayacucho, las familias a menudo también «circularán» a los niños, enviándolos a vivir con familiares en otras partes del departamento como una forma de ayudarlos a recibir una educación que no está disponible en el pueblo y para ayudar al pariente a recibir la ayuda del niño. Esto no requiere inversión financiera per se, aunque es explícitamente económico, como plantea Leinaweaver. Anteriormente sugirió la circulación infantil como una alternativa a las esterilizaciones masivas (2008, pp. 13-14); sin embargo, de manera menos drástica, también podría ser una solución a la limitación infantil basada solo en preocupaciones económicas. Esta es otra consideración omitida en el discurso del centro de salud.

Ello sugiere que la narrativa financiera dominante se ve favorecida no solo porque es la única preocupación real: hay otras alternativas muy reales, como se discutió anteriormente. Sin embargo, es favorecida porque logra un dinero final específico, que es lo único que las mujeres indígenas pobres no tienen. Cuando se convierten en madres, son llevadas al mundo de la asistencia social del Estado, donde el gobierno se interesa de manera inmediata e invasiva por su situación económica. Pero, además, es un punto de entrada donde el Estado puede manipular su reclamo de ciudadanía a través de sus hijos y su «deuda» y el deber con el gobierno (pobre).

La maternidad hace que una mujer sea visible a través de su nueva dependencia de la atención médica del SIS y Juntos, y como dice Bridges, «las madres pobres están radicalmente incapacitadas por su capacidad de ser vistas por el Estado» (2017, p. 87). Como ha argumentado este documento, debido a que la toma de decisiones aparentemente irresponsable llevó a las mujeres a quedar embarazadas 
mientras eran pobres, se les revocó su reclamo de ciudadanía plena hasta el momento en que pudieran dar a luz con éxito niños profesionales competentes, étnica, cultural y racialmente «superiores» a su madre indigente, en opinión del Estado. Finalmente, como sugiere Ames (2013), el Estado peruano necesita considerar e incorporar el imaginario social en sus políticas hacia el desarrollo rural. En este caso, se recomienda prestar atención a las experiencias de las mujeres quechuas, y en especial de las madres. En la actualidad, si uno es pobre e indígena en la zona rural de Ayacucho, su maternidad condiciona la ciudadanía.

\section{REFERENCIAS}

Ames, Patricia (2013). Educación, desarrollo y desigualdad. En B. Revesz (ed.), Miradas cruzadas: políticas públicas y desarrollo regional en el Perú (pp. 147-175). Lima: IEP.

Ballón, A. (2014). Introducción. En A. Ballón (ed.), Memorias del caso peruano de esterilización forzada (pp. 27-46). Lima: Biblioteca Nacional del Perú.

Barrig, M. (2001). El mundo al revés: imágenes de la mujer indígena. Buenos Aires: CLACSO.

Bridges, K. (2011). Reproducing Race: An Ethnography of Pregnancy as a Site of Racialization. California: University of California. https://doi.org/10.1525/california/9780520268944.001.0001

Bridges, K. (2017). The Poverty of Privacy Rights. California: Stanford.

Cavero Carrasco, R. (2008). La educación para indígenas en los Andes peruanos: una mirada intercultural (1941-1989). Cuadernos Interculturales, 6(11), 46-71.

Calisaya, E. (2004). Voces de las mujeres de Ancash: género y salud reproductiva. Lima: Movimiento Manuela Ramos.

Chirapaq (2017). ¿Es útil ser indígena? Identidad, censos y políticas públicas. Lima: Chirapaq.

Condori, F. L. (2003). Voces de mujeres de Huancavelica: género y salud reproductiva. Lima: Movimiento Manuela Ramos.

Crivello, G. 2011. «Becoming Somebody»: youth transitions through education and migration in Peru'. Journal of Youth Studies, 14(4), 395-411. https://doi.org/10.1 $080 / 13676261.2010 .538043$

Estrada Alarcón, Luz (2004). Voces de las mujeres quechuas y aymaras de Puno: género y salud reproductiva. Lima: Movimiento Manuela Ramos. 
Ewig, Christina (2010). Second-Wave Neoliberalism: Gender, Race, and Health Sector Reform in Peru. University Park, PA: Pennsylvania State University Press.

Ewig, Christina (2014). La economía política de las esterilizaciones forzadas en el Perú. En Alejandra Ballón (ed.), Memorias del caso peruano de esterilización forzada. Lima: Biblioteca Nacional del Perú.

García, María Elena (2010). Rethinking BilingualEducation in Peru: Intercultural Politics, State Policy and Indigenous Rights. International Journal of Bilingual Education and Bilingualism, 7(5), 348-367. https://doi.org/10.1080/13670050408667819

García, María Elena (2005). Making Indigenous Citizens: Identities, Education and Multicultural Development in Peru. Palo Alto, CA: Stanford University Press

Gillespie, Bronwen (2016). Much More than Malnutrition: Motherhood and the State in the Peruvian Andes. Doctoral Thesis. Sussex: University of Sussex.

Goldberg, David Theo (2015). Are we all postracial yet? (Debating Race). Cambridge: Polity.

Harvey, David (2007). A Brief History of Neoliberalism. Nueva York: Oxford.

La República (2016). Hijos y nietos de víctimas del terrorismo podrán acceder a Beca 18. 6 de febrero de 2016. https://larepublica.pe/sociedad/739288-familiares-directos-de-victimas-del-terrorismo-podran-acceder-beca-18 Accessed 01/12/2018.

Leinaweaver, Jessaca (2009). Los niños ayacuchanos: una antropología de la adopción y la construcción familiar en el Perú. Lima: IEP.

Leinaweaver, Jessaca (2008). Mass Sterilizations and Child Circulation in Peru. Anthropology News, 46(1), 13-14. https://doi.org/10.1525/an.2005.46.1.13

Meltzer, J. (2013). «Good citizenship» and the promotion of personal savings accounts in Peru. Citizenship Studies, 17(5), 641-652. https://doi.org/10.1080/13621025. 2013.818382

Molyneux, Maxine y Marilyn Thomson (2011). Cash transfers, gender equity and women's empowerment in Peru, Ecuador and Bolivia. Gender and Development, 19(2), 195-212. https://doi.org/10.1080/13552074.2011.592631

Necochea López, R. (2016). La planificación familiar en el Perú del siglo XX. Lima: IEP.

Ramírez Hita, Susana (2009). La contribución del método etnográfico al registro del dato epidemiológico. Epidemiología sociocultural indígena quechua de la ciudad de Potosí. Salud Colectiva, 5(1), 63-85. https://doi.org/10.18294/sc.2009.231

Ramírez Hita, Susana (2013). Usos y desusos del método etnográfico. Las limitaciones de las narrativas en el campo de la salud. En O. Romani (ed.), Etnografia, metodologías cualitativas e investigación en salud: un debate abierto. Tarragona: URV. 
Rapp, Rayna (1999). Testing Women, Testing the Fetus: The Social Impact of Amniocentesis in America. Nueva York: Routledge.

Roberts, Elizabeth (2012a). God's Laboratory: Assisted Reproduction in the Andes. Berkeley, CA: University of Berkeley.

Roberts, Elizabeth (2012b). Scars of a Nation: Surgical Penetration and the Ecuadorian State. The Journal of Latin American and Caribbean Anthropology, 17(3), 215-237. https://doi.org/10.1111/j.1935-4940.2012.01223.x

Salcedo, Celine (2004). Voces de mujeres de Ayacucho: género y salud reproductiva. Lima: Movimiento Manuela Ramos.

Tamayo, Gulia (2014). De entuertos y a tuerto: las verdades incómodas del PNSRPF, la renuencia a investigar judicialmente crímenes sobre el derecho internacional y sus consecuencias sobre las víctimas. En Alejandra Ballón (ed.), Memorias del caso peruano de esterilización forzada. Lima: Biblioteca Nacional del Perú.

Tubino, Fidel y Roberto Zariquiey (2007). Jenetian. El juego de las identidades en tiempos de lluvia. Lima: UNMSM.

Weismantel, M. Jeanne (1992). Food, Gender and Poverty in the Ecuadorian Andes. Pennsylvania: University of Pennsylvania.

Yon Leau, C. (2000). Hablan las mujeres andinas: preferencias reproductivas y anticoncepción. Lima: Movimiento Manuela Ramos. 